(c) 2006 International Press

Adv. Theor. Math. Phys. 10 (2006) 261-282

\title{
On the relation between ADM and Bondi energy-momenta
}

\author{
Xiao Zhang \\ Institute of Mathematics, Academy of Mathematics and Systems Science, \\ Chinese Academy of Sciences, Beijing 100080, China \\ xzhang@amss.ac.cn
}

\begin{abstract}
When a spacetime takes Bondi radiating metric and is vacuum and asymptotically flat at spatial infinity which ensures the positive mass theorem, we prove that the standard ADM energy-momentum is the past limit of the Bondi energy-momentum. We also derive a formula relating the ADM energy-momentum of any asymptotically flat spacelike hypersurface to the Bondi energy-momentum of any null hypersurface. The formula indicates that the Bondi mass is always less than the ADM total energy if the system has news.

The assumed asymptotic flatness precludes gravitational radiation. We therefore study further the relation between the ADM total energy and the Bondi mass when gravitational radiation emits. We find that, in this case, the ADM total energy is no longer the past limit of the Bondi mass. They differ by certain quantity relating to the news of the system.
\end{abstract}

\section{Introduction}

It is well known that the ADM total energy and total linear momentum can be defined on asymptotically flat spatial infinity in a spacetime [1].

e-print archive: http://lanl.arXiv.org/abs/gr-qc/0511036 
The fundamental positive mass conjecture in general relativity, which was proved by Schoen-Yau, and later by Witten, asserts that the ADM energymomentum is always timelike for a non-trivial spacetime [18-20, 25]. In [26], the positive mass theorem was extended to the case involving the total angular momentum. This extension actually relates to the Einstein-Cartan theory. On the other hand, in the pioneering work of Bondi and coworkers $[5,17,24]$ on the gravitational waves in vacuum spacetimes, the Bondi mass associated to each null cone is defined and their main result asserts this Bondi mass is always non-increasing with respect to the retarded time.

One main problem in general relativity is to understand what exactly happens on the energy-momentum when an asymptotically flat spacelike hypersurface goes to a null hypersurface. Physically, it is believed that gravitational radiation occurs, and the energy of the system will be carried away by gravitational waves. When a spacetime can be conformally compactified, and asymptotically empty and flat at null and spatial infinity in the sense of [2], Ashtekar and Magnon-Ashtekar demonstrated the mass at spatial infinity is the past limit of the Bondi mass taken as the cut approaches the "point" of spatial infinity [3]. This result was later verified, in the framework of Penrose, by Hayward replacing the Penrose conformal factor by a product of advanced and retarded conformal factors [11] and by Valiente Kroon [10, 22, 23] using a representation of spatial infinity based on the properties of conformal geodesics. In [6], Christodoulou and Klainerman proved the global existence of globally hyperbolic, strongly asymptotically flat, maximal foliated vacuum Einstein equations and proved rigorously that the ADM mass at spatial infinity is the past limit of the Bondi mass. In this paper, we assume that spacetime takes vacuum Bondi radiating metric. We define the spatial infinity as the "real" time slice. Under the asymptotic flatness conditions at spatial infinity which ensures the positive mass theorem, we use a complete and rigorous argument to demonstrate that the standard ADM energy-momentum is the past limit of the "standard" Bondi energy-momentum defined in $[5,17]$. We derive a formula relating the ADM energy-momentum for a spacelike hypersurface at time $t_{0}$ to the Bondi energy-momentum for a null hypersurface at retarded time $u_{0}$. As a consequence, we prove that the Bondi mass is always less than the ADM total energy if the system has news.

However, it is presumably believed that the assumptions of asymptotic flatness at spatial infinity in all above works preclude gravitational radiation, at least near spatial infinity. We therefore assume certain weaker conditions on asymptotic flatness at spatial infinity which spacetimes may include gravitational radiation. We also derive a formula relating the ADM total energy to the Bondi mass. We find that, in this case, the ADM total 
RELATION BETWEEN ADM AND BONDI ENERGY-MOMENTA 263

energy is no longer the past limit of the Bondi mass and it differs by certain quantity relating to the news of the system.

It should be pointed out that the "real" time $t$ is assumed to be the retarded time $u$ plus the Euclidean distance $r$ in the paper. This condition is very restricted, which is not satisfied even in Schwartzschild spacetime. In the forthcoming paper [12], we will study the more general case which $t$ approaches to $u+r$ in certain sense.

The paper is organized as follows: In Section 2, we state some well-known formulation and results of Bondi, van der Burg, Metzner and Sachs. In Section 3, we give some asymptotically flat conditions on Bondi radiating metric, which ensures the ADM total energy is well defined at spatial infinity. In Section 4, we derive the second fundamental form of spatial infinity and show that it is also asymptotically flat, which ensures the ADM total linear momentum is well defined at spatial infinity. In Section 5, we prove that the ADM total energy is the Bondi mass of negatively infinite retarded time. In Section 6, we prove that the ADM total linear momentum is the Bondi momentum of negatively infinite retarded time. In Section 7 , we establish a relation between the ADM total energy-momentum of spatial infinity at any time and the Bondi energy-momentum at any retarded time. We also prove that the Bondi mass is always less than the ADM total energy if the system has news. In Section 8, we establish a relation between the ADM total energy and the Bondi mass for Bondi radiating metric which includes gravitational radiation.

\section{The Bondi coordinates}

Throughout the paper, we assume that $\left(L^{3,1}, \tilde{g}\right)$ is a vacuum spacetime with metric $\tilde{g}=\tilde{g}_{i j} d x^{i} d x^{j}$ taking the following Bondi radiating metric

$$
\begin{aligned}
\tilde{g}= & -\left(-\frac{V}{r} \mathrm{e}^{2 \beta}+r^{2} \mathrm{e}^{2 \gamma} U^{2} \cosh 2 \delta+r^{2} \mathrm{e}^{-2 \gamma} W^{2} \cosh 2 \delta\right. \\
& \left.+2 r^{2} U W \sinh 2 \delta\right) d u^{2}-2 \mathrm{e}^{2 \beta} d u d r \\
& -2 r^{2}\left(\mathrm{e}^{2 \gamma} U \cosh 2 \delta+W \sinh 2 \delta\right) d u d \theta \\
& -2 r^{2}\left(\mathrm{e}^{-2 \gamma} W \cosh 2 \delta+U \sinh 2 \delta\right) \sin \theta d u d \psi \\
& +r^{2}\left(\mathrm{e}^{2 \gamma} \cosh 2 \delta d \theta^{2}+\mathrm{e}^{-2 \gamma} \cosh 2 \delta \sin ^{2} \theta d \psi^{2}+2 \sinh 2 \delta \sin \theta d \theta d \psi\right)
\end{aligned}
$$


and satisfies the outgoing radiation condition, where $\beta, \gamma, \delta, U, V, W$ are functions of

$$
x^{0}=u, \quad x^{1}=r, \quad x^{2}=\theta, \quad x^{3}=\psi .
$$

$u$ is a retarded coordinate, $r$ is Euclidean distance, $\theta$ and $\psi$ are spherical coordinates, $0 \leq \theta \leq \pi, 0 \leq \psi \leq 2 \pi$. We assume the "real" time $t$ is defined as

$$
t=u+r .
$$

In the forthcoming paper [12], we will study the more general relation between the "real" time $t$ and the retarded time $u$. The metric (2.1) was studied by Bondi and coworkers [5, 17, 24] in the theory of gravitational waves in general relativity. They proved that the following asymptotic behavior holds

$$
\begin{aligned}
\gamma & =\frac{c(u, \theta, \psi)}{r}+\mathrm{O}\left(\frac{1}{r^{3}}\right), \\
\delta & =\frac{d(u, \theta, \psi)}{r}+\mathrm{O}\left(\frac{1}{r^{3}}\right), \\
\beta & =-\frac{c^{2}+d^{2}}{4 r^{2}}+\mathrm{O}\left(\frac{1}{r^{4}}\right), \\
U & =-\frac{l(u, \theta, \psi)}{r^{2}}+\mathrm{O}\left(\frac{1}{r^{3}}\right), \\
W & =-\frac{\bar{l}(u, \theta, \psi)}{r^{2}}+\mathrm{O}\left(\frac{1}{r^{3}}\right), \\
V & =-r+2 M(u, \theta, \psi)+\mathrm{O}\left(\frac{1}{r}\right),
\end{aligned}
$$

where

$$
\begin{aligned}
& l=c_{, 2}+2 c \cot \theta+d_{, 3} \csc \theta, \\
& \bar{l}=d_{, 2}+2 d \cot \theta-c_{, 3} \csc \theta .
\end{aligned}
$$

(Throughout the paper, denote $f_{, i}=\frac{\partial f}{\partial x^{i}}$ for $i=0,1,2,3$.) $M$ is the mass aspect and $c_{, 0}, d_{, 0}$ are the news functions and they satisfy the following equation [24]:

$$
M_{, 0}=-\left(\left(c_{, 0}\right)^{2}+\left(d_{, 0}\right)^{2}\right)+\frac{1}{2}\left(l_{, 2}+l \cot \theta+\bar{l}_{, 3} \csc \theta\right)_{, 0} .
$$

To avoid the singularity, we assume

Condition A. Each of the six functions $\beta, \gamma, \delta, U, V, W$ together with its derivatives up to the second orders are equal at $\psi=0$ and $2 \pi$. 
RELATION BETWEEN ADM AND BONDI ENERGY-MOMENTA 265

This implies

$$
\begin{gathered}
\left.M\right|_{\psi=0}=\left.M\right|_{\psi=2 \pi},\left.\quad M_{, p}\right|_{\psi=0}=\left.M_{, p}\right|_{\psi=2 \pi}, \\
\left.c\right|_{\psi=0}=\left.c\right|_{\psi=2 \pi},\left.\quad c_{, p}\right|_{\psi=0}=\left.c_{, p}\right|_{\psi=2 \pi}, \\
\left.d\right|_{\psi=0}=\left.d\right|_{\psi=2 \pi},\left.\quad d_{, p}\right|_{\psi=0}=\left.d_{, p}\right|_{\psi=2 \pi}
\end{gathered}
$$

for $p, q=0,2,3$. Denote $S^{2}$ the unit 2-sphere. The physical reason requires [5]

Condition B. For all $u$,

$$
\int_{0}^{2 \pi} c(u, 0, \psi) d \psi=0, \quad \int_{0}^{2 \pi} c(u, \pi, \psi) d \psi=0
$$

Let $N_{u_{0}}$ be a null hypersurface which is given by $u=u_{0}$ at null infinity. The Bondi energy-momentum of $N_{u_{0}}$ is defined by $[5,8]$

$$
m_{\nu}\left(u_{0}\right)=\frac{1}{4 \pi} \int_{S^{2}} M\left(u_{0}, \theta, \psi\right) n^{\nu} d S
$$

where $\nu=0,1,2,3, n^{0}=1, n^{i}$ the restriction of the natural coordinate $x^{i}$ to the unit round sphere, i.e.,

$$
n^{0}=1, \quad n^{1}=\sin \theta \cos \psi, \quad n^{2}=\sin \theta \sin \psi, \quad n^{3}=\cos \theta .
$$

$m_{0}$ is referred as the Bondi mass. Under Condition A and Condition B,

$$
\begin{aligned}
\int_{S^{2}}\left(l_{, 2}+l \cot \theta+\bar{l}_{, 3} \csc \theta\right) d S= & \int_{0}^{\pi} \int_{0}^{2 \pi}\left(l_{, 2} \sin \theta+l \cos \theta+\bar{l}_{, 3}\right) d \psi d \theta \\
= & \left.\int_{0}^{2 \pi}(l \sin \theta)\right|_{\theta=0} ^{\pi} d \psi+\int_{0}^{\pi}(\bar{l}(u, \theta, 2 \pi) \\
& -\bar{l}(u, \theta, 0)) d \theta \\
= & -2 \int_{0}^{2 \pi}(c(u, \pi, \psi)+c(u, 0, \psi)) d \psi \\
= & 0,
\end{aligned}
$$

then (2.2) gives rise to the famous Bondi mass loss formula

$$
\frac{d}{d u} m_{0}=-\frac{1}{4 \pi} \int_{S^{2}}\left((c, 0)^{2}+(d, 0)^{2}\right) d S .
$$


Now we derive the Bondi momentum loss formula. It is easy to find

$$
\begin{aligned}
\int_{S^{2}} & \left(l_{, 2}+l \cot \theta+\bar{l}_{, 3} \csc \theta\right) n^{1} d S \\
= & \int_{0}^{\pi} \int_{0}^{2 \pi}\left(l, 2 \sin \theta+l \cos \theta+\bar{l}_{, 3}\right) \sin \theta \cos \psi d \psi d \theta \\
= & \int_{0}^{2 \pi}\left(\left.\left(l \sin ^{2} \theta\right)\right|_{\theta=0} ^{\pi}-\int_{0}^{\pi} l \sin \theta \cos \theta d \theta\right) \cos \psi d \psi \\
& +\int_{0}^{\pi}\left(\left.(\bar{l} \cos \psi)\right|_{\psi=0} ^{2 \pi}+\int_{0}^{2 \pi} \bar{l} \sin \psi d \psi\right) \sin \theta d \theta \\
= & -\int_{0}^{\pi} \int_{0}^{2 \pi}(c \cos \psi+d \cos \theta \sin \psi) d \psi d \theta \\
& +\int_{0}^{\pi} \int_{0}^{2 \pi}(c \cos \psi+d \cos \theta \sin \psi) d \psi d \theta \\
= & 0,
\end{aligned}
$$

and

$$
\begin{aligned}
\int_{S^{2}} & \left(l_{, 2}+l \cot \theta+\bar{l}_{, 3} \csc \theta\right) n^{2} d S \\
= & \int_{0}^{\pi} \int_{0}^{2 \pi}\left(l_{, 2} \sin \theta+l \cos \theta+\bar{l}_{, 3}\right) \sin \theta \sin \psi d \psi d \theta \\
= & \int_{0}^{2 \pi}\left(\left.\left(l \sin ^{2} \theta\right)\right|_{\theta=0} ^{\pi}-\int_{0}^{\pi} l \sin \theta \cos \theta d \theta\right) \sin \psi d \psi \\
& +\int_{0}^{\pi}\left(\left.(\bar{l} \sin \psi)\right|_{\psi=0} ^{2 \pi}-\int_{0}^{2 \pi} \bar{l} \cos \psi d \psi\right) \sin \theta d \theta \\
= & -\int_{0}^{\pi} \int_{0}^{2 \pi}(c \sin \psi-d \cos \theta \cos \psi) d \psi d \theta \\
& +\int_{0}^{\pi} \int_{0}^{2 \pi}(c \sin \psi-d \cos \theta \cos \psi) d \psi d \theta \\
= & 0,
\end{aligned}
$$

and

$$
\begin{aligned}
\int_{S^{2}} & \left(l_{, 2}+l \cot \theta+\bar{l}_{, 3} \csc \theta\right) n^{3} d S \\
= & \int_{0}^{\pi} \int_{0}^{2 \pi}\left(l_{, 2} \sin \theta+l \cos \theta+\bar{l}_{, 3}\right) \cos \theta d \psi d \theta \\
= & \int_{0}^{\pi} \int_{0}^{2 \pi}\left(l \sin ^{2} \theta\right) d \psi d \theta+\left.\int_{0}^{2 \pi}(l \sin \theta \cos \theta)\right|_{\theta=0} ^{\pi} d \psi
\end{aligned}
$$




$$
\begin{aligned}
& =2 \int_{0}^{2 \pi}(c(u, \pi, \psi)-c(u, 0, \psi)) d \psi \\
& =0
\end{aligned}
$$

We obtain, for $k=1,2,3$,

$$
\frac{d}{d u} m_{k}=-\frac{1}{4 \pi} \int_{S^{2}}\left(\left(c_{, 0}\right)^{2}+\left(d_{, 0}\right)^{2}\right) n^{k} d S
$$

In general, the spatial infinity in vacuum spacetimes which the metric satisfies (2.1) may not be asymptotically flat in the sense of [14, 18-20, 25, $26]$. Using $(t, r, \theta, \psi)$ coordinates, the metric (2.1) can be written as

$$
\begin{aligned}
\tilde{g}= & -\left(-\frac{V}{r} \mathrm{e}^{2 \beta}+r^{2} \mathrm{e}^{2 \gamma} U^{2} \cosh 2 \delta\right. \\
& \left.+r^{2} \mathrm{e}^{-2 \gamma} W^{2} \cosh 2 \delta+2 r^{2} U W \sinh 2 \delta\right) d t^{2} \\
& -2\left(\left(1+\frac{V}{r}\right) \mathrm{e}^{2 \beta}-r^{2} \mathrm{e}^{2 \gamma} U^{2} \cosh 2 \delta\right. \\
& \left.-r^{2} \mathrm{e}^{-2 \gamma} W^{2} \cosh 2 \delta-2 r^{2} U W \sinh 2 \delta\right) d t d r \\
& -2 r^{2}\left(\mathrm{e}^{2 \gamma} U \cosh 2 \delta+W \sinh 2 \delta\right) d t d \theta \\
& -2 r^{2}\left(\mathrm{e}^{-2 \gamma} W \cosh 2 \delta+U \sinh 2 \delta\right) \sin \theta d t d \psi \\
& +\left(\left(2+\frac{V}{r}\right) \mathrm{e}^{2 \beta}-r^{2} \mathrm{e}^{2 \gamma} U^{2} \cosh 2 \delta\right. \\
& \left.-r^{2} \mathrm{e}^{-2 \gamma} W^{2} \cosh 2 \delta-2 r^{2} U W \sinh 2 \delta\right) d r^{2} \\
& +r^{2}\left(\mathrm{e}^{2 \gamma} \cosh 2 \delta d \theta^{2}+\mathrm{e}^{-2 \gamma} \cosh 2 \delta \sin { }^{2} \theta d \psi^{2}\right. \\
& \left.+2 \sinh ^{2} \sin \theta d \theta d \psi\right) \\
& +2 r^{2}\left(\mathrm{e}^{2 \gamma} U \cosh 2 \delta+W \sinh 2 \delta\right) d r d \theta \\
& +2 r^{2}\left(\mathrm{e}^{-2 \gamma} W \cosh 2 \delta+U \sinh 2 \delta\right) \sin \theta d r d \psi
\end{aligned}
$$




\section{The spatial infinity}

Let $\left(N_{t_{0}}, g, h\right)$ be a spacelike hypersurface in $L^{3,1}$ which is given by $\left\{t=t_{0}\right\}$, where $g$ is the induced metric of $\tilde{g}$ and $h$ is the second fundamental form.

$$
\begin{aligned}
g= & \left(\left(2+\frac{V}{r}\right) \mathrm{e}^{2 \beta}-r^{2} \mathrm{e}^{2 \gamma} U^{2} \cosh 2 \delta\right. \\
& \left.-r^{2} \mathrm{e}^{-2 \gamma} W^{2} \cosh 2 \delta-2 r^{2} U W \sinh 2 \delta\right) d r^{2} \\
& +r^{2}\left(\mathrm{e}^{2 \gamma} \cosh 2 \delta d \theta^{2}+\mathrm{e}^{-2 \gamma} \cosh 2 \delta \sin ^{2} \theta d \psi^{2}\right. \\
& +2 \sinh 2 \delta \sin \theta d \theta d \psi) \\
& +2 r^{2}\left(\mathrm{e}^{2 \gamma} U \cosh 2 \delta+W \sinh 2 \delta\right) d r d \theta \\
& +2 r^{2}\left(\mathrm{e}^{-2 \gamma} W \cosh 2 \delta+U \sinh 2 \delta\right) \sin \theta d r d \psi
\end{aligned}
$$

$\left(N_{t_{0}}, g, h\right)$ is usually refereed to an initial data set. We will study when $\left(N_{t_{0}}, g, h\right)$ is asymptotically flat. Let $\left\{\breve{e}^{i}\right\}(i=1,2,3)$ be the coframe of the standard flat metric $g_{0}$ on $\mathbb{R}^{3}$,

$$
\breve{e}^{1}=d r, \quad \breve{e}^{2}=r d \theta, \quad \breve{e}^{3}=r \sin \theta d \psi .
$$

Let $\left\{\breve{e}_{i}\right\} \quad(i=1,2,3)$ be the dual frame. The connection 1 -form $\left\{\breve{\omega}_{i j}\right\}$ is given by $d \breve{e}^{i}=-\breve{\omega}_{i j} \wedge \breve{e}^{j}$, or $\breve{\nabla} \breve{e}_{i}=-\breve{\omega}_{i j} \otimes \breve{e}_{j}(i, j=1,2,3)$, where $\breve{\nabla}$ is LeviCivita connection of $g_{0}$. It is easy to find that

$$
\breve{\omega}_{12}=-\frac{1}{r} \breve{e}^{2}, \quad \breve{\omega}_{13}=-\frac{1}{r} \breve{e}^{3}, \quad \breve{\omega}_{23}=-\frac{\cot \theta}{r} \breve{e}^{3} .
$$

Throughout the paper, we denote $\breve{\nabla}_{i} \equiv \breve{\nabla}_{\breve{e}_{i}}$ for $i=1,2,3$.

The initial data set $\left(N_{t_{0}}, g, h\right)$ is asymptotically flat in the current case if the metric $g$ satisfies

$$
\begin{aligned}
& g\left(\breve{e}_{i}, \breve{e}_{j}\right)=\delta_{i j}+\mathrm{O}\left(\frac{1}{r}\right), \breve{\nabla}_{k} g\left(\breve{e}_{i}, \breve{e}_{j}\right)=\mathrm{O}\left(\frac{1}{r^{2}}\right), \\
& \breve{\nabla}_{l} \breve{\nabla}_{k} g\left(\breve{e}_{i}, \breve{e}_{j}\right)=\mathrm{O}\left(\frac{1}{r^{3}}\right)
\end{aligned}
$$

as $r \rightarrow \infty$. Furthermore, 2-tensor $h$ satisfies

$$
h\left(\breve{e}_{i}, \breve{e}_{j}\right)=\mathrm{O}\left(\frac{1}{r^{2}}\right), \quad \breve{\nabla}_{k} h\left(\breve{e}_{i}, \breve{e}_{j}\right)=\mathrm{O}\left(\frac{1}{r^{3}}\right)
$$

as $r \rightarrow \infty$. 
RELATION BETWEEN ADM AND BONDI ENERGY-MOMENTA 269

Denote $\mathcal{C}_{\left\{a_{1}, a_{2}, a_{3}\right\}}$ the functions in spacetime which satisfy the following asymptotic behavior at spatial infinity

$$
\mathcal{C}_{\left\{a_{1}, a_{2}, a_{3}\right\}}=\left\{\begin{aligned}
\lim _{r \rightarrow \infty u \rightarrow-\infty} r^{a_{1}} f & =\mathrm{O}(1), \\
f: \lim _{r \rightarrow \infty u \rightarrow-\infty} r^{a_{2}} \breve{\nabla}_{i} f & =\mathrm{O}(1), \\
\lim _{r \rightarrow \infty} \lim _{u \rightarrow-\infty} r^{a_{3}} \breve{\nabla}_{i} \breve{\nabla}_{j} f & =\mathrm{O}(1)
\end{aligned}\right\} .
$$

We employ the following assumptions:

Condition C. $\gamma \in \mathcal{C}_{\{1,2,3\}}, \delta \in \mathcal{C}_{\{1,2,3\}}, \beta \in \mathcal{C}_{\{2,3,4\}}, U \in \mathcal{C}_{\{2,3,4\}}, W \in \mathcal{C}_{\{2,3,4\}}$, $V+r \in \mathcal{C}_{\{0,1,2\}}$.

Condition $\mathrm{C}$ implies, for $r$ sufficiently large,

$$
\begin{aligned}
& \lim _{u \rightarrow-\infty} M=\mathrm{O}(1), \quad \lim _{u \rightarrow-\infty} c=\mathrm{O}(1), \quad \lim _{u \rightarrow-\infty} d=\mathrm{O}(1), \\
& \lim _{u \rightarrow-\infty} M_{, 0}=\mathrm{O}\left(\frac{1}{r}\right), \quad \lim _{u \rightarrow-\infty} c_{, 0}=\mathrm{O}\left(\frac{1}{r}\right), \quad \lim _{u \rightarrow-\infty} d_{, 0}=\mathrm{O}\left(\frac{1}{r}\right), \\
& \lim _{u \rightarrow-\infty} M_{, A}=\mathrm{O}(1), \quad \lim _{u \rightarrow-\infty} c_{, A}=\mathrm{O}(1), \quad \lim _{u \rightarrow-\infty} d_{, A}=\mathrm{O}(1),
\end{aligned}
$$

where $A, B=2,3$.

Proposition 3.1. Under Condition A, Condition B, and Condition $C$, the metric $g$ of $N_{t_{0}}$ satisfies (3.2).

Proof. The components of the metric $g$ are

$$
\begin{aligned}
g\left(\breve{e}_{1}, \breve{e}_{1}\right)= & {\left[\left(2+\frac{V}{r}\right) \mathrm{e}^{2 \beta}-r^{2} \mathrm{e}^{2 \gamma} U^{2} \cosh 2 \delta\right.} \\
& \left.-r^{2} \mathrm{e}^{-2 \gamma} W^{2} \cosh 2 \delta-2 r^{2} U W \sinh 2 \delta\right]_{t=t_{0}} \\
g\left(\breve{e}_{2}, \breve{e}_{2}\right)= & \left.\mathrm{e}^{2 \gamma} \cosh 2 \delta\right|_{t=t_{0}} \\
g\left(\breve{e}_{3}, \breve{e}_{3}\right)= & \left.\mathrm{e}^{-2 \gamma} \cosh 2 \delta\right|_{t=t_{0}} \\
g\left(\breve{e}_{1}, \breve{e}_{2}\right)= & r\left(\mathrm{e}^{2 \gamma} U \cosh 2 \delta+W \sinh 2 \delta\right)_{t=t_{0}} \\
g\left(\breve{e}_{1}, \breve{e}_{3}\right)= & r\left(\mathrm{e}^{-2 \gamma} W \cosh 2 \delta+U \sinh 2 \delta\right)_{t=t_{0}} \\
g\left(\breve{e}_{2}, \breve{e}_{3}\right)= & \left.\sinh 2 \delta\right|_{t=t_{0}} .
\end{aligned}
$$

Note that for fixed $t=t_{0}, r \rightarrow \infty$ is equivalent to $u \rightarrow-\infty$, a straightforward computation yields the proposition. 


\section{The second fundamental form}

The lapse $\mathcal{N}$ and the shift $X_{i}(i=1,2,3)$ of the spacelike hypersurface $N_{t_{0}}$ are

$$
\begin{aligned}
& \mathcal{N}=\left(-\tilde{g}^{t t}\right)_{t=t_{0}}^{-1 / 2}, \\
& X_{i}=\left.\tilde{g}_{t i}\right|_{t=t_{0}} .
\end{aligned}
$$

The second fundamental form is then given by

$$
h_{i j}=\frac{1}{2 \mathcal{N}}\left(\nabla_{i} X_{j}+\nabla_{j} X_{i}-\partial_{t} \tilde{g}_{i j}\right)
$$

where

$$
\nabla_{i} X_{j}=\partial_{i} X_{j}-\Gamma_{i j}^{k} X_{k}
$$

and

$$
\Gamma_{i j}^{k}=\frac{1}{2} g^{k l}\left(\frac{\partial g_{l i}}{\partial x^{j}}+\frac{\partial g_{l j}}{\partial x^{i}}-\frac{\partial g_{i j}}{\partial x^{l}}\right)_{t=t_{0}}
$$

are Christoffel symbols of the metric $g$. Now we compute the inverse $g^{i j}$ of metric tensor $g_{i j}$ of $N_{t_{0}}$. Denote $\bar{g}=\left(g_{A B}\right), 2 \leq A, B \leq 3$, i.e.,

$$
\bar{g}=r^{2}\left(\begin{array}{cc}
\mathrm{e}^{2 \gamma} \cosh 2 \delta & \sinh 2 \delta \sin \theta \\
\sinh 2 \delta \sin \theta & \mathrm{e}^{-2 \gamma} \cosh 2 \delta \sin ^{2} \theta
\end{array}\right) .
$$

Then, the inverse $\bar{g}^{-1}=\left(g^{A B}\right)$

$$
\bar{g}^{-1}=\frac{1}{r^{2}}\left(\begin{array}{cc}
\mathrm{e}^{-2 \gamma} \cosh 2 \delta & -\frac{\sinh 2 \delta}{\sin \theta} \\
-\frac{\sinh 2 \delta}{\sin \theta} & \mathrm{e}^{2 \gamma} \frac{\cosh 2 \delta}{\sin ^{2} \theta}
\end{array}\right) .
$$

Using the formulae [7]

$$
\begin{aligned}
\frac{1}{g^{11}} & =g_{11}-\bar{g}^{A B} g_{1 B} g_{1 A}, \\
\frac{g^{1 A}}{g^{11}} & =-\bar{g}^{A B} g_{1 B}, \\
g^{A B} & =\bar{g}^{A B}+\frac{g_{1 A} g_{1 B}}{g^{11}},
\end{aligned}
$$


we obtain

$$
\begin{aligned}
\frac{1}{g^{11}}= & \left(2+\frac{V}{r}\right) \mathrm{e}^{2 \beta}-2 r^{2} \mathrm{e}^{2 \gamma} U^{2} \cosh 2 \delta \\
& -2 r^{2} \mathrm{e}^{-2 \gamma} W^{2} \cosh 2 \delta-4 r^{2} U W \sinh 2 \delta, \\
g^{12}= & -U g^{11}, \\
g^{13}= & -\frac{W}{\sin \theta} g^{11}, \\
g^{22}= & \frac{\mathrm{e}^{-2 \gamma} \cosh 2 \delta}{r^{2}}+U^{2} g^{11}, \\
g^{23}= & -\frac{\sinh 2 \delta}{r^{2} \sin \theta}+\frac{U W}{\sin \theta} g^{11}, \\
g^{33}= & \frac{\mathrm{e}^{2 \gamma} \cosh 2 \delta}{r^{2} \sin ^{2} \theta}+\frac{W^{2}}{\sin ^{2} \theta} g^{11} .
\end{aligned}
$$

Proposition 4.1. Under Condition A, Condition B and Condition $C$, the second fundamental form $h$ of $N_{t_{0}}$ satisfies (3.3).

Proof. With the help of asymptotic behavior of $\beta, \gamma, \delta, U, V, W$, we obtain the asymptotic expansion of the Christoffel symbols of (3.1) (see Appendix). And a straightforward computation yields

$$
\begin{aligned}
\mathcal{N}^{2}= & -\tilde{g}_{t t}+\tilde{g}_{t i} \tilde{g}_{t j} g^{i j} \\
= & \mathrm{e}^{4 \beta} g^{11} \\
= & 1-\left.\frac{2 M}{r}\right|_{t=t_{0}}+\mathrm{O}\left(\frac{1}{r^{2}}\right) . \\
X_{1}= & -\left(1+\frac{V}{r}\right) \mathrm{e}^{2 \beta}+r^{2} \mathrm{e}^{2 \gamma} U^{2} \cosh 2 \delta \\
& +r^{2} \mathrm{e}^{-2 \gamma} W^{2} \cosh 2 \delta+2 r^{2} U W \sinh 2 \delta \\
= & -\left.\frac{2 M}{r}\right|_{t=t_{0}}+\mathrm{O}\left(\frac{1}{r^{2}}\right), \\
X_{2}= & -r^{2}\left(\mathrm{e}^{2 \gamma} U \cosh 2 \delta+W \sinh 2 \delta\right) \\
= & \left.l\right|_{t=t_{0}}+\mathrm{O}\left(\frac{1}{r}\right), \\
X_{3}= & -r^{2}\left(\mathrm{e}^{-2 \gamma} W \cosh 2 \delta+U \sinh 2 \delta\right) \sin \theta \\
= & \bar{l}_{\sin \theta}+\mathrm{O}\left(\frac{1}{r}\right) .
\end{aligned}
$$


We obtain the second fundamental forms

$$
\begin{aligned}
h_{11} & =\left.\frac{2 M+r M_{, 0}}{r^{2}}\right|_{t=t_{0}}+\mathrm{O}\left(\frac{1}{r^{3}}\right), \\
h_{22} & =\left(l_{, 2}-2 M-r c_{, 0}\right)_{t=t_{0}}+\mathrm{O}\left(\frac{1}{r}\right), \\
h_{33} & =\left(\bar{l}_{, 3} \sin \theta-2 M \sin ^{2} \theta+l \sin \theta \cos \theta+r c_{, 0} \sin ^{2} \theta\right)_{t=t_{0}}+\mathrm{O}\left(\frac{1}{r}\right), \\
h_{12} & =-\left.\frac{M_{, 2}+l}{r}\right|_{t=t_{0}}+\mathrm{O}\left(\frac{1}{r^{2}}\right), \\
h_{13} & =-\left.\frac{M_{, 3}+l \sin \theta}{r}\right|_{t=t_{0}}+\mathrm{O}\left(\frac{1}{r^{2}}\right), \\
h_{23} & =\left(\frac{\bar{l}_{, 2} \sin \theta-\bar{l} \cos \theta+l_{, 3}}{2}-r d_{, 0} \sin \theta\right)_{t=t_{0}}+\mathrm{O}\left(\frac{1}{r}\right) .
\end{aligned}
$$

Note that for fixed $t=t_{0}, r \rightarrow \infty$ is equivalent to $u \rightarrow-\infty$, a straightforward computation yields the proposition.

The trace of the second fundamental form is

$$
\operatorname{tr}_{g}(h)=\frac{1}{r^{2}}\left(r M_{, 0}-2 M+l \cot \theta+l_{, 2}+\bar{l}_{, 3} \csc \theta\right)_{t=t_{0}}+\mathrm{O}\left(\frac{1}{r^{3}}\right) .
$$

\section{The ADM total energy}

In this section, we compute the ADM total energy of $N_{t_{0}}$. In polar coordinates, the ADM total energy $\mathbb{E}$ is

$$
\mathbb{E}=\frac{1}{16 \pi} \lim _{r \rightarrow \infty} \int_{S_{r}}\left(\breve{\nabla}^{j} g\left(\breve{e}_{1}, \breve{e}_{j}\right)-\breve{\nabla}_{1} \operatorname{tr}_{g_{0}}(g)\right) \breve{e}^{2} \wedge \breve{e}^{3} .
$$

This can be seen by changing Euclidean coordinates to the polar coordinates or by writing Witten's mass formula $[14,25,26]$ in asymptotically polar coordinates and using the comparison of two spin connections [28]. The polar coordinate expression of the ADM total energy is equivalent to the Euclidean coordinate expression of [1] even if the metric is not the standard asymptotically flat in the sense of $[14,18-20,25,26]$. This is because the coordinate transformation relates to only the ground metric, which is the standard metric, of $\mathbb{R}^{3}$.

Theorem 5.1. Under Condition A, Condition B, and Condition $C$, the ADM total energy of $N_{t_{0}}$ is

$$
\mathbb{E}\left(t_{0}\right)=m_{0}(-\infty)
$$


Proof. By (3.1), we obtain

$$
\begin{aligned}
\breve{\nabla}^{j} g\left(\breve{e}_{1}, \breve{e}_{j}\right)-\breve{\nabla}_{1} \operatorname{tr}_{g_{0}}(g)= & \breve{e}_{j}\left(g\left(\breve{e}_{1}, \breve{e}_{j}\right)\right)-\breve{e}_{1} \operatorname{tr}_{g_{0}}(g) \\
& -g\left(\breve{e}_{j}, \breve{e}_{i}\right) \breve{\omega}_{i 1}\left(\breve{e}_{j}\right) \\
& -g\left(\breve{e}_{1}, \breve{e}_{i}\right) \breve{\omega}_{i j}\left(\breve{e}_{j}\right) \\
= & \frac{4 M}{r^{2}}-\frac{l_{, 2}}{r^{2}}-\frac{l \cot \theta}{r^{2}}-\frac{\breve{l}_{, 3}}{r^{2} \sin \theta} \\
& +\mathrm{O}\left(\frac{1}{r^{3}}\right) .
\end{aligned}
$$

Thus,

$$
\begin{aligned}
\mathbb{E}\left(t_{0}\right)= & \left.\frac{1}{4 \pi} \lim _{r \rightarrow \infty} \int_{S^{2}} M\right|_{t=t_{0}} d S \\
& -\frac{1}{16 \pi} \lim _{r \rightarrow \infty} \int_{0}^{\pi} \int_{0}^{2 \pi}\left(l, 2 \sin \theta+l \cos \theta+\bar{l}_{, 3}\right)_{t=t_{0}} d \psi d \theta \\
= & \frac{1}{4 \pi} \lim _{u \rightarrow-\infty} \int_{S^{2}} M d S \\
& -\frac{1}{16 \pi} \lim _{u \rightarrow-\infty} \int_{0}^{\pi}(\bar{l}(u, \theta, 2 \pi)-\bar{l}(u, \theta, 0)) d \theta \\
& -\left.\frac{1}{16 \pi} \lim _{u \rightarrow-\infty} \int_{0}^{2 \pi}(l \sin \theta)\right|_{\theta=0} ^{\pi} d \psi \\
= & \frac{1}{4 \pi} \lim _{u \rightarrow-\infty} \int_{S^{2}} M(u, \theta, \psi) d S \\
& +\frac{1}{8 \pi} \lim _{u \rightarrow-\infty} \int_{0}^{2 \pi}(c(u, 0, \psi)+c(u, \pi, \psi)) d \psi \\
= & m_{0}(-\infty) .
\end{aligned}
$$

\section{The ADM total linear momentum}

In this section, we compute the ADM total linear momentum of $N_{t_{0}}$. Let Euclidean coordinates

$$
y^{1}=r \sin \theta \cos \psi, \quad y^{2}=r \sin \theta \sin \psi, \quad y^{3}=r \cos \theta .
$$

Then, the ADM total linear momentum

$$
\mathbb{P}_{i}=\frac{1}{8 \pi} \lim _{r \rightarrow \infty} \int_{S_{r}}\left(h\left(\frac{\partial}{\partial y^{i}}, \frac{\partial}{\partial r}\right)-g\left(\frac{\partial}{\partial y^{i}}, \frac{\partial}{\partial r}\right) \operatorname{tr}_{g}(h)\right) \breve{e}^{2} \wedge \breve{e}^{3} .
$$


A simple computation yields

$$
\begin{aligned}
\frac{\partial}{\partial y^{1}} & =\frac{\partial}{\partial r} n^{1}+\frac{\partial}{\partial \theta} \frac{\cos \theta \cos \psi}{r}-\frac{\partial}{\partial \psi} \frac{\sin \psi}{r \sin \theta} \\
\frac{\partial}{\partial y^{2}} & =\frac{\partial}{\partial r} n^{2}+\frac{\partial}{\partial \theta} \frac{\cos \theta \sin \psi}{r}+\frac{\partial}{\partial \psi} \frac{\cos \psi}{r \sin \theta} \\
\frac{\partial}{\partial y^{3}} & =\frac{\partial}{\partial r} n^{3}-\frac{\partial}{\partial \theta} \frac{\sin \theta}{r}
\end{aligned}
$$

Therefore, under Condition A, Condition B, and Condition C,

$$
\begin{aligned}
h\left(\frac{\partial}{\partial y^{1}}, \frac{\partial}{\partial r}\right)= & h_{11} n^{1}+h_{21} \frac{\cos \theta \cos \psi}{r}-h_{31} \frac{\sin \psi}{r \sin \theta} \\
= & \frac{1}{r^{2}}\left(\left(2 M+r M_{, 0}\right) n^{1}-\left(M_{, 2}+l\right) \cos \theta \cos \psi\right. \\
& \left.+\frac{\sin \psi}{\sin \theta}\left(M_{, 3}+\bar{l} \sin \theta\right)\right)+\mathrm{O}\left(\frac{1}{r^{3}}\right), \\
h\left(\frac{\partial}{\partial y^{2}}, \frac{\partial}{\partial r}\right)= & h_{11} n^{2}+h_{21} \frac{\cos \theta \sin \psi}{r}+h_{31} \frac{\cos \psi}{r \sin \theta} \\
= & \frac{1}{r^{2}}\left(\left(2 M+r M_{, 0}\right) n^{2}-\left(M_{, 2}+l\right) \cos \theta \sin \psi\right. \\
& -\frac{\cos \psi}{\sin \theta}\left(M_{, 3}+\bar{l} \sin \theta\right)+\mathrm{O}\left(\frac{1}{r^{3}}\right), \\
h\left(\frac{\partial}{\partial y^{3}}, \frac{\partial}{\partial r}\right)= & h_{11} n^{3}-h_{21} \frac{\sin \theta}{r} \\
= & \frac{1}{r^{2}}\left(\left(2 M+r M_{, 0}\right) n^{3}+\left(M_{, 2}+l\right) \sin \theta\right)+\mathrm{O}\left(\frac{1}{r^{3}}\right) . \\
g\left(\frac{\partial}{\partial y^{1}}, \frac{\partial}{\partial r}\right)= & g_{11} n^{1}+g_{21} \frac{\cos \theta \cos \psi}{r}-g_{31} \frac{\sin \psi}{r \sin \theta} \\
g\left(\frac{\partial}{\partial y^{2}}, \frac{\partial}{\partial r}\right)= & g_{11} n^{2}+g_{21} \frac{\cos \theta \sin \psi}{r}+g_{31} \frac{\cos \psi}{r \sin \theta} \\
= & \left(1+\frac{2 M}{r}\right) n^{2}-l \frac{\cos \theta \sin \psi}{r}-\bar{l} \frac{\cos \psi}{r}+\mathrm{O}\left(\frac{1}{r^{2}}\right),
\end{aligned}
$$




$$
\begin{aligned}
g\left(\frac{\partial}{\partial y^{3}}, \frac{\partial}{\partial r}\right) & =g_{11} n^{3}-g_{21} \frac{\sin \theta}{r} \\
& =\left(1+\frac{2 M}{r}\right) n^{3}+l \frac{\sin \theta}{r}+\mathrm{O}\left(\frac{1}{r^{2}}\right) .
\end{aligned}
$$

Theorem 6.1. Under Condition A, Condition $B$ and Condition $C$, the $A D M$ total linear momentum of $N_{t_{0}}$ is

$$
\mathbb{P}_{k}\left(t_{0}\right)=m_{k}(-\infty)
$$

for $k=1,2,3$.

Proof. It is straightforward that

$$
\begin{aligned}
\mathbb{P}_{1}\left(t_{0}\right)= & \frac{1}{2 \pi} \lim _{u \rightarrow-\infty} \int_{S^{2}} M n^{1} d S \\
& -\frac{1}{8 \pi} \lim _{u \rightarrow-\infty} \int_{0}^{\pi} \int_{0}^{2 \pi}\left(M_{, 2} \sin \theta \cos \theta \cos \psi-M_{, 3} \sin \psi\right) d \psi d \theta \\
& -\frac{1}{8 \pi} \lim _{u \rightarrow-\infty} \int_{0}^{\pi} \int_{0}^{2 \pi}\left(l_{, 2} \sin ^{2} \theta \cos \psi+\bar{l}_{, 3} \sin \theta \cos \psi\right) d \psi d \theta \\
& -\frac{1}{8 \pi} \lim _{u \rightarrow-\infty} \int_{0}^{\pi} \int_{0}^{2 \pi}(2 l \sin \theta \cos \theta \cos \psi-\bar{l} \sin \theta \sin \psi) d \psi d \theta \\
= & \frac{1}{4 \pi} \lim _{u \rightarrow-\infty} \int_{S^{2}} M n^{1} d S-\left.\frac{1}{8 \pi} \lim _{u \rightarrow-\infty} \int_{0}^{\pi}(\bar{l} \sin \theta \cos \psi)\right|_{\psi=0} ^{2 \pi} d \theta \\
& -\left.\frac{1}{8 \pi} \lim _{u \rightarrow-\infty} \int_{0}^{2 \pi}\left(l \sin ^{2} \theta \cos \psi\right)\right|_{\theta=0} ^{\pi} d \psi \\
= & \frac{1}{4 \pi} \lim _{u \rightarrow-\infty} \int_{S^{2}} M n^{1} d S \\
= & m_{1}(-\infty),
\end{aligned}
$$

and

$$
\begin{aligned}
\mathbb{P}_{2}\left(t_{0}\right)= & \frac{1}{2 \pi} \lim _{u \rightarrow-\infty} \int_{S^{2}} M n^{2} d S \\
& -\frac{1}{8 \pi} \lim _{u \rightarrow-\infty} \int_{0}^{\pi} \int_{0}^{2 \pi}\left(M_{, 2} \sin \theta \cos \theta \sin \psi+M_{, 3} \cos \psi\right) d \psi d \theta \\
& -\frac{1}{8 \pi} \lim _{u \rightarrow-\infty} \int_{0}^{\pi} \int_{0}^{2 \pi}\left(l_{, 2} \sin ^{2} \theta \sin \psi+\bar{l}_{, 3} \sin \theta \sin \psi\right) d \psi d \theta \\
& -\frac{1}{8 \pi} \lim _{u \rightarrow-\infty} \int_{0}^{\pi} \int_{0}^{2 \pi}(2 l \sin \theta \cos \theta \sin \psi+\bar{l} \sin \theta \cos \psi) d \psi d \theta
\end{aligned}
$$




$$
\begin{aligned}
= & \frac{1}{4 \pi} \lim _{u \rightarrow-\infty} \int_{S^{2}} M n^{2} d S-\left.\frac{1}{8 \pi} \lim _{u \rightarrow-\infty} \int_{0}^{\pi}(\bar{l} \sin \theta \sin \psi)\right|_{\psi=0} ^{2 \pi} d \theta \\
& -\left.\frac{1}{8 \pi} \lim _{u \rightarrow-\infty} \int_{0}^{2 \pi} \psi\left(l \sin ^{2} \theta \sin \psi\right)\right|_{\theta=0} ^{\pi} d \psi \\
= & \frac{1}{4 \pi} \lim _{u \rightarrow-\infty} \int_{S^{2}} M n^{2} d S \\
= & m_{2}(-\infty),
\end{aligned}
$$

and

$$
\begin{aligned}
\mathbb{P}_{3}\left(t_{0}\right)= & \frac{1}{2 \pi} \lim _{u \rightarrow-\infty} \int_{S^{2}} M n^{3} d S \\
& +\frac{1}{8 \pi} \lim _{u \rightarrow-\infty} \int_{0}^{\pi} \int_{0}^{2 \pi}\left(M_{, 2} \sin ^{2} \theta\right) d \psi d \theta \\
& -\frac{1}{8 \pi} \lim _{u \rightarrow-\infty} \int_{0}^{\pi} \int_{0}^{2 \pi}\left(l_{, 2} \sin \theta \cos \theta+\bar{l}_{, 3} \cos \theta\right) d \psi d \theta \\
& +\frac{1}{8 \pi} \lim _{u \rightarrow-\infty} \int_{0}^{\pi} \int_{0}^{2 \pi}\left(l \sin ^{2} \theta-l \cos ^{2} \theta\right) d \psi d \theta \\
= & \frac{1}{4 \pi} \lim _{u \rightarrow-\infty} \int_{S^{2}} M n^{3} d S-\left.\frac{1}{8 \pi} \lim _{u \rightarrow-\infty} \int_{0}^{2 \pi}(l \sin \theta \cos \theta)\right|_{\theta=0} ^{\pi} d \psi \\
= & \frac{1}{4 \pi} \lim _{u \rightarrow-\infty} \int_{S^{2}} M n^{3} d S \\
& -\frac{1}{4 \pi} \lim _{u \rightarrow-\infty} \int_{0}^{2 \pi}(c(u, \pi, \psi)-c(u, 0, \psi)) d \psi \\
= & m_{3}(-\infty) .
\end{aligned}
$$

\section{$7 \quad \mathrm{ADM}$ and Bondi energy-momenta}

In this section, we derive a formula relating the ADM total energy and total linear momentum for a spacelike hypersurface at time $t_{0}$ to the Bondi energy-momentum for a null hypersurface at retarded time $u_{0}$ in non-radiative fields.

Theorem 7.1. Under Condition A, Condition B, and Condition $C$, the $A D M$ total energy, the ADM total linear momentum of $N_{t_{0}}$ and the Bondi energy-momentum of null hypersurface $N_{u_{0}}$ satisfy

$$
\mathbb{P}_{\nu}\left(t_{0}\right)=m_{\nu}\left(u_{0}\right)+\frac{1}{4 \pi} \int_{-\infty}^{u_{0}} \int_{S^{2}}\left(\left(c_{, 0}\right)^{2}+(d, 0)^{2}\right) n^{\nu} d S d u
$$


RELATION BETWEEN ADM AND BONDI ENERGY-MOMENTA 277

for $\nu=0,1,2,3$, and $\mathbb{E}\left(t_{0}\right)$ is denoted as $\mathbb{P}_{0}\left(t_{0}\right)$. In particular, if there is news, then the ADM total energy is always greater than the Bondi mass.

Proof. By (2.2), we obtain

$$
\begin{aligned}
M\left(u_{0}\right)= & \lim _{u \rightarrow-\infty} M(u)+\int_{-\infty}^{u_{0}} M_{, 0} d u \\
= & \lim _{u \rightarrow-\infty} M(u)+\int_{-\infty}^{u_{0}}\left(\left(c_{, 0}\right)^{2}+\left(d_{, 0}\right)^{2}\right) d u \\
& +\left.\frac{1}{2}\left(l_{, 2}+l \cot \theta+\bar{l}_{, 3} \csc \theta\right)\right|_{u=-\infty} ^{u_{0}} .
\end{aligned}
$$

Then the theorem is a direct consequence of (2.3), (2.4), Theorem 5.1 and Theorem 6.1.

Remark 7.1. If we do not further assume Condition B, then

$$
\begin{aligned}
\mathbb{E}\left(t_{0}\right)= & m_{0}\left(u_{0}\right)+\frac{1}{4 \pi} \int_{-\infty}^{u_{0}} \int_{S^{2}}\left((c, 0)^{2}+\left(d_{, 0}\right)^{2}\right) d S d u \\
& +\frac{1}{4 \pi} \int_{0}^{2 \pi}\left(c\left(u_{0}, 0, \psi\right)+c\left(u_{0}, \pi, \psi\right)\right) d \psi \\
& -\frac{1}{8 \pi} \int_{0}^{2 \pi}(c(-\infty, 0, \psi)+c(-\infty, \pi, \psi)) d \psi, \\
\mathbb{P}_{1}\left(t_{0}\right)= & m_{1}\left(u_{0}\right)+\frac{1}{4 \pi} \int_{-\infty}^{u_{0}} \int_{S^{2}}\left(\left(c_{, 0}\right)^{2}+\left(d_{, 0}\right)^{2}\right) n^{1} d S d u, \\
\mathbb{P}_{2}\left(t_{0}\right)= & m_{2}\left(u_{0}\right)+\frac{1}{4 \pi} \int_{-\infty}^{u_{0}} \int_{S^{2}}\left((c, 0)^{2}+\left(d_{, 0}\right)^{2}\right) n^{2} d S d u, \\
\mathbb{P}_{3}\left(t_{0}\right)= & m_{3}\left(u_{0}\right)+\frac{1}{4 \pi} \int_{-\infty}^{u_{0}} \int_{S^{2}}\left(\left(c_{, 0}\right)^{2}+\left(d_{, 0}\right)^{2}\right) n^{3} d S d u \\
& +\frac{1}{4 \pi} \int_{0}^{2 \pi}\left(c\left(u_{0}, 0, \psi\right)-c\left(u_{0}, \pi, \psi\right)\right) d \psi .
\end{aligned}
$$

Remark 7.2. If the spacetime $L^{3,1}$ satisfies the dominant energy condition, then the positive mass theorem $[18-20,25]$

$$
\mathbb{E} \geq\left(\mathbb{P}_{1}^{2}+\mathbb{P}_{2}^{2}+\mathbb{P}_{3}^{2}\right)^{1 / 2}
$$

and Theorem 7.1 give rise to an inequality involving the Bondi energymomentum. 


\section{Radiative fields}

It is a fundamental problem to find appropriate conditions of asymptotically flatness at spatial infinity to include gravitational radiation. Suggested by the Sommerfeld electromagnetic boundary conditions, Trautman [21] specified a class of (non-covariant) boundary conditions for a spatially confined gravitational source. The covariant formulation of the Trautman boundary conditions was given by Papadopoulos and Witten [15].

The Bondi metric (2.1) gives rise to a class of asymptotic flatness also. Instead of Condition $\mathrm{C}$, we assume the metric has the following asymptotic behavior at spatial infinity.

Condition D. $\gamma \in \mathcal{C}_{\{1,1,1\}}, \quad \delta \in \mathcal{C}_{\{1,1,1\}}, \beta \in \mathcal{C}_{\{2,2,2\}}, U \in \mathcal{C}_{\{2,2,2\}}, W \in$
$\quad \mathcal{C}_{\{2,2,2\}}, V+r \in \mathcal{C}_{\{0,0,0\}}$.

Condition D implies, for $r$ sufficiently large,

$$
\begin{aligned}
& \lim _{u \rightarrow-\infty} M=\mathrm{O}(1), \quad \lim _{u \rightarrow-\infty} c=\mathrm{O}(1), \quad \lim _{u \rightarrow-\infty} d=\mathrm{O}(1) \\
& \lim _{u \rightarrow-\infty} M_{, 0}=\mathrm{O}(1), \quad \lim _{u \rightarrow-\infty} c_{, 0}=\mathrm{O}(1), \quad \lim _{u \rightarrow-\infty} d_{, 0}=\mathrm{O}(1), \\
& \lim _{u \rightarrow-\infty} M_{, A}=\mathrm{O}(1), \quad \lim _{u \rightarrow-\infty} c_{, A}=\mathrm{O}(1), \quad \lim _{u \rightarrow-\infty} d_{, A}=\mathrm{O}(1),
\end{aligned}
$$

where $A, B=2,3$. Physically, Condition $\mathrm{D}$ might be an interpretation of Sommerfeld's radiation condition at spatial infinity: In [5], the authors found, in axi-symmetric spacetime, this condition imply that $\gamma=\frac{f_{1}(t-r)}{r}+$ $\frac{f_{2}(t-r)}{r}+\cdots$ as $r \rightarrow \infty$. As the metric (2.1) behaves as a "wave", we may think that $f_{1}, f_{2}, \ldots$ involve $\sin (t-r), \cos (t-r)$, etc. This is essential for our motivation to introduce Condition D.

Now we derive a formula between the ADM total energy and the Bondi mass. This may be thought as the relation between them in radiative fields. The relation between the ADM total linear momentum $P_{k}$ and the Bondi momentum $m_{k}$ in radiative fields requires much more dedicated computation. This question will be addressed elsewhere.

Theorem 8.1. Let $\mathbb{E}\left(t_{0}\right)$ be the ADM total energy of spacelike hypersurface $N_{t_{0}}$ whose metric satisfies (3.1). Under Condition A, Condition B, and Condition D, we have

$$
\mathbb{E}\left(t_{0}\right)=m_{0}(-\infty)+\frac{1}{4 \pi} \lim _{u \rightarrow-\infty} \int_{0}^{\pi} \int_{0}^{2 \pi}\left(c^{2}+d^{2}\right)_{, 0} \sin \theta d \psi d \theta
$$


Proof. Note that (3.1) gives

$$
\begin{aligned}
g\left(\breve{e}_{2}, \breve{e}_{2}\right)+g\left(\breve{e}_{3}, \breve{e}_{3}\right) & =2 \cosh 2 \gamma \cosh 2 \delta \\
& =2+4\left(\gamma^{2}+\delta^{2}\right)+\mathrm{O}\left(\frac{1}{r^{4}}\right) \\
& =2+\frac{4\left(c^{2}+d^{2}\right)}{r^{2}}+\mathrm{O}\left(\frac{1}{r^{3}}\right) .
\end{aligned}
$$

Hence, as $r \rightarrow \infty$ (or $u \rightarrow-\infty)$,

$$
\begin{aligned}
\breve{\nabla}^{j} g\left(\breve{e}_{1}, \breve{e}_{j}\right)-\breve{\nabla}_{1} \operatorname{tr}_{g_{0}}(g)= & \breve{e}_{j}\left(g\left(\breve{e}_{1}, \breve{e}_{j}\right)\right)-\breve{e}_{1} \operatorname{tr}_{g_{0}}(g) \\
& -g\left(\breve{e}_{j}, \breve{e}_{i}\right) \breve{\omega}_{i 1}\left(\breve{e}_{j}\right)-g\left(\breve{e}_{1}, \breve{e}_{i}\right) \breve{\omega}_{i j}\left(\breve{e}_{j}\right) \\
= & \frac{4 M}{r^{2}}-\frac{l_{, 2}}{r^{2}}-\frac{l \cot \theta}{r^{2}}-\frac{\bar{l}_{, 3}}{r^{2} \sin \theta} \\
& -4 \breve{e}_{1}\left(\frac{c^{2}+d^{2}}{r^{2}}\right)+\mathrm{O}\left(\frac{1}{r^{3}}\right) \\
= & \frac{4 M}{r^{2}}-\frac{l_{, 2}}{r^{2}}-\frac{l \cot \theta}{r^{2}}-\frac{\bar{l}_{, 3}}{r^{2} \sin \theta} \\
& +\frac{4\left(c^{2}+d^{2}\right)_{, 0}}{r^{2}}+\mathrm{O}\left(\frac{1}{r^{3}}\right) .
\end{aligned}
$$

This gives rise to Theorem 8.1.

\section{Appendix}

The asymptotic expansion of the Christoffel symbols of (3.1) under Condition A, Condition B, and Condition C:

$$
\begin{aligned}
\Gamma_{11}^{1} & =-\frac{M+r M_{, 0}}{r^{2}}+\mathrm{O}\left(\frac{1}{r^{3}}\right), \\
\Gamma_{12}^{1} & =\frac{l+M_{, 2}}{r}+\mathrm{O}\left(\frac{1}{r^{2}}\right), \\
\Gamma_{13}^{1} & =\frac{l \sin \theta+M_{, 3}}{r}+\mathrm{O}\left(\frac{1}{r^{2}}\right), \\
\Gamma_{22}^{1} & =-r+\mathrm{O}(1), \\
\Gamma_{23}^{1} & =\frac{1}{2}\left(-\bar{l}_{, 2} \sin \theta+\bar{l} \cos \theta+l_{, 3}-2 d \sin \theta+2 r d_{, 0} \sin \theta\right)+\mathrm{O}\left(\frac{1}{r}\right), \\
\Gamma_{33}^{1} & =-r \sin ^{2} \theta+\mathrm{O}(1),
\end{aligned}
$$




$$
\begin{aligned}
& \Gamma_{11}^{2}=-\frac{M_{, 2}-r l_{, 0}}{r^{3}}+\mathrm{O}\left(\frac{1}{r^{4}}\right), \\
& \Gamma_{12}^{2}=\frac{1}{r}+\mathrm{O}\left(\frac{1}{r^{2}}\right), \\
& \Gamma_{13}^{2}=-\frac{-l_{, 3}+\left(\bar{l}_{\sin \theta}\right)_{, 2}-2\left(d+r d_{, 0}\right) \sin \theta}{2 r^{2}}+\mathrm{O}\left(\frac{1}{r^{3}}\right), \\
& \Gamma_{22}^{2}=-\frac{l-c_{, 2}}{r}+\mathrm{O}\left(\frac{1}{r^{2}}\right), \\
& \Gamma_{23}^{2}=-\frac{2 d \cos \theta-c_{, 3}}{r}+\mathrm{O}\left(\frac{1}{r^{2}}\right), \\
& \Gamma_{33}^{2}=-\sin \theta \cos \theta+\mathrm{O}\left(\frac{1}{r}\right), \\
& \Gamma_{11}^{3}=-\frac{M_{, 3}-r \bar{l}_{0}}{r^{3} \sin { }^{2} \theta}+\mathrm{O}\left(\frac{1}{r^{4}}\right), \\
& \Gamma_{12}^{3}=-\frac{-l_{, 3}+\left(\bar{l}_{\sin } \theta\right)_{, 2}+2 r d_{, 0}}{2 r^{2} \sin ^{2} \theta}+\mathrm{O}\left(\frac{1}{r^{3}}\right), \\
& \Gamma_{13}^{3}=\frac{1}{r}+\mathrm{O}\left(\frac{1}{r^{2}}\right), \\
& \Gamma_{22}^{3}=-\frac{\bar{l}_{\sin \theta}-2 d_{, 2} \sin _{33} \theta-2 d \cos \theta+c_{, 3}}{r \sin ^{2} \theta}+\mathrm{O}\left(\frac{1}{r^{2}}\right), \\
& \Gamma_{23}^{3}=\frac{\cos \theta}{\sin \theta}+\mathrm{O}\left(\frac{1}{r}\right), \\
& \Gamma_{\operatorname{lin} \theta+c_{, 3}-2 d \cos \theta}^{3}+\mathrm{O}\left(\frac{1}{r^{2}}\right) .
\end{aligned}
$$

\section{Acknowledgments}

The author is indebted to Yun Kau Lau for pointing out to him the works of Ashtekar and Magnon-Ashtekar, Rizzi and Hayward and many useful conversations and to Wenling Huang for her careful reading of the manuscript and many valuable suggestions. This work was partially done when the author visited the Center of Mathematical Sciences, Zhejiang University and he would like to thank the center for its hospitality. The research is partially supported by National Natural Science Foundation of China under grant no. 10231050 and the Innovation Project of Chinese Academy of Sciences. 
RELATION BETWEEN ADM AND BONDI ENERGY-MOMENTA 281

\section{References}

[1] S. Arnowitt, S. Deser and C. Misner, Coordinate invariance and energy expressions in general relativity, Phys. Rev. 122 (1961), 997-1006.

[2] A. Ashtekar and R. Hansen, A unified treatment of null and spatial infinity in general relativity. I. Universal structure, asymptotic symmetries, and conserved quantities at spatial infinity, J. Math. Phys. 19 (1978), 1542-1566.

[3] A. Ashtekar and A. Magnon-Ashtekar, Energy-momentum in general relativity, Phys. Rev. Lett. 43 (1979), 181-184.

[4] R. Bartnik, Bondi mass in the NQS gauge, Class. Quantum Grav. 21 (2004), S59-S71.

[5] H. Bondi, M. van der Burg and A. Metzner, Gravitational waves in general relativity VII. Waves from axi-symmetric isolated systems, Proc. Roy. Soc. London A 269 (1962), 21-52.

[6] D. Christodoulou and S. Klainerman, The global nonlinear stablity of Minkowski space, Princeton Math. Series 41, Princeton Univ. Press, Princeton, 1993.

[7] P. Chruściel, J. Jezierski and J. Kijowski, Hamiltonian field theory in the radiating regime, Lect. Notes Physics, Monographs, 70, SpringerVerlag, Berlin, 2002.

[8] P. Chruściel, J. Jezierski and M. MacCallum, Uniqueness of the Trautman-Bondi mass, Phys. Rev. D 58 (1998), 084001-084016.

[9] P. Chruściel, M. MacCallum and D. Singleton, Gravitational waves in general relativity. XIV. Bondi expansions and the "polyhomogeneity" of Scr I. Philos. Trans. Roy. Soc. London A 350 (1995), 113-141.

[10] H. Friedrich and J. Kannar, Bondi-type systems near space-time infinity and the calculation of the NP-constants, J. Math. Phys. 41 (2000), 2195-2232.

[11] S. Hayward, Spatial and null infinity via advanced and retarded conformal factors, Phys. Rev. D 68 (2003), 104015.

[12] W.-I. Huang and X. Zhang, On the relation between ADM and Bondi energy-momenta III - perturbed radiative spatial infinity, gr-qc/0601022.

[13] R. Penrose and W. Rindler, Spinors and space-time, I, II, Cambridge University Press, 1984, 1988.

[14] T. Parker and C. Taubes, On Witten's proof of the positive energy theorem, Commun. Math. Phys. 84 (1982), 223-238.

[15] D. Papadopoulos and L. Witten, Covariant boundary conditions in gravitational radiation theory: a new covariant definition of 
spatially asymptotically flat spacetimes, Phys. Rev. D 23 (1981), 267-271.

[16] A. Rizzi, Angular momentum in general relativity: the definition at null infinity includes the spatial definition as a special case, Phys. Rev. D 63 (2001), 104002.

[17] R. Sachs, Gravitational waves in general relativity VIII. Waves in asymptotically flat space-time, Proc. Roy. Soc. London, A 270 (1962), 103-126.

[18] R. Schoen and S.T. Yau, On the proof of the positive mass conjecture in general relativity, Commun. Math. Phys. 65 (1979), 45-76.

[19] R. Schoen and S.T. Yau, The energy and the linear momentum of spacetimes in general relativity, Commun. Math. Phys. 79 (1981), 47-51.

[20] R. Schoen and S.T. Yau, Proof of the positive mass theorem. II, Commun. Math. Phys. 79 (1981), 231-260.

[21] A. Trautman, Radiation and boundary conditions in the theory of gravitation, Bull. Acad. Pol. Sci., Ser. Sci. Math. VI 407 (1958) 407-412.

[22] J. Valiente Kroon, Early radiative properties of the developments of time-symmetric conformally flat initial data, Class. Quantum Grav. 20 (2003) L53-L59.

[23] J. Valiente Kroon, Does asymptotic simplicity allow for radiation near spatial infinity, Commun. Math. Phys. 251 (2004), 211-234.

[24] M. van der Burg, Gravitational waves in general relativity IX. Conserved quantities, Proc. Roy. Soc. London A 294 (1966), 112-122.

[25] E. Witten, A new proof of the positive energy theorem, Commun. Math. Phys. 80 (1981), 381-402.

[26] X. Zhang, Angular momentum and positive mass theorem, Commun. Math. Phys. 206 (1999), 137-155.

[27] X. Zhang, Remarks on the total angular momentum in general relativity, Commun. Theor. Phys. 39 (2003), 521-524.

[28] X. Zhang, A definition of total energy-momenta and the positive mass theorem on asymptotically hyperbolic 3-manifolds I, Commun. Math. Phys. 249 (2004), 529-548. 